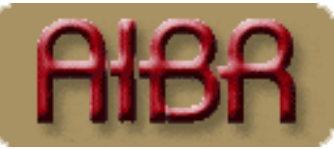

REVISTA DE ANTROPOLOGÍA IBEROAMERICANA

ESTIMADO LECTOR/A:

GRACIAS POR DESCARGAR ESTE ARTÍCULO. EL TEXTO QUE ESTÁ A PUNTO DE CONSULTAR ES DE ACCESO LIBRE Y GRATUITO GRACIAS AL TRABAJO Y LA COLABORACIÓN DESINTERESADA DE UN AMPLIO COLECTIVO DE PROFESIONALES.

USTED PUEDE AYUDARNOS A INCREMENTAR LA CALIDAD Y A MANTENER LA LIBRE DIFUSIÓN DE LOS CONTENIDOS DE ESTA REVISTA A TRAVÉS DE SU INSCRIPCIÓN A LA ASOCIACIÓN AIBR:

http://www.aibr.org/antropologia/aibr/socios.php

La asociación a AIBR tiene un coste mínimo al año, y le proporcionará las siguientes ventajas y privilegios:

1. Recibir en su domicilio la revista impresa, en Europa y América (tres números anuales), así como todas las novedades relativas al funcionamiento de la asociación.

2. Recibir en su domicilio, a precio especial o de forma gratuita, cuantas publicaciones adicionales edite la asociación.

3. Derecho a voto en las asambleas de socios, así como a presentarse como candidato a la elección de su Junta Directiva.

4. Recibir el boletín de socios (tres números anuales), así como la información económica relativa a cuentas anuales de la asociación.

5. Beneficiarse de las reducciones de precio en congresos, cursos, libros y todos aquellos convenios a los que a nivel corporativo AIBR llegue con otras entidades. En este momento, existen los siguientes acuerdos:

o Reducción de un $20 \%$ en el precio de todos los libros publicados por la editorial MELUSINA.

o Reducción de un $20 \%$ en el precio de todos los libros publicados por la editorial SEPHA.

o Reducción de un 30\% en el precio de todos los libros publicados por la editorial GRAN VÍA.

o Derecho a cuota reducida en los congresos trianuales de la FAAEE (España) y a los bianuales de la Sociedad Española de Antropología Aplicada.

o Derecho a cuota reducida en los congresos la IUAES.

6. Promoción gratuita, tanto a través de la revista electrónica como de la revista impresa, de aquellas publicaciones de las que sea autor y que estén registradas con ISBN. La difusión se realiza entre más de 5.000 antropólogos suscritos a la revista.

7. Cuenta de correo electrónico ilimitada de la forma socio@aibr.org, para consultar a través de webmail o cualquier programa externo.

8. Espacio para web personal de la forma http://www.aibr.org/(directorio)/(nombre) y cuenta propia de ftp.

9. Acceso con clave a todos los documentos de la Intranet de socios de AIBR, incluida la consulta a artículos en proceso de evaluación de la revista AIBR.

10. Promoción gratuita a través de la revista (banner rotativo y reseña) de aquellos eventos, congresos, conferencias o cursos en los que usted forme parte del comité organizador.

11. Opción a formar parte como evaluador de los artículos recibidos por la revista.

IMPORTE DE LA CUOTA ANUAL: Hasta diciembre de 2008, la cuota única anual es de 32 (euros). Su validez es de un año a partir del pago de la cuota. Por favor, revise la actualización de cuotas en nuestra web. 


\title{
AlB universalidad Y VARIabilidad cultural www.aibr.org DE LA RISA Y EL HUMOR
}

\section{Eduardo Jáuregui}

Saint Louis University (Campus de Madrid). E-mail: eduardo@humorpositivo.com

\begin{abstract}
Resumen
La risa es un fenómeno aparentemente universal, pero el humor que la provoca muestra una variabilidad casi infinita. Este artículo trata de distinguir entre los aspectos universales de la risa y los que son culturalmente variables. Define la risa como una emoción básica del ser humano, provocada por un estímulo cognitivo que depende de definiciones sociales de la situación, lo cual explica al menos en parte su aparente heterogeneidad. Otros aspectos que también pueden variar son las normas y costumbres relativas a la expresión de la risa y del humor, los géneros humorísticos, las instituciones del humor y las definiciones culturales de la risa misma.
\end{abstract}

Palabras clave
Risa, humor, emociones.

Abstract

Laughter is an apparently universal phenomenon, but the humor that causes it seems almost infinitely varied. This article attempts to distinguish between universal aspects of laughter and those which are subject to the observable cultural variability. It defines laughter as a basic human emotion, caused by a cognitive stimulus influenced by social definitions, which explains at least in part its apparent heterogeneity. Other variable aspects include the norms and customs associated with the display of laughter and humor, the various humor genres and formats, humorous institutions and the cultural definitions of laughter itself.

Key words

Laughter, humor, emotions.

Recibido: 20 de Septiembre de 2007

Aceptado: 15 de Diciembre de 2007

\section{Introducción}

- a risa es un fenómeno que lleva milenios fascinando al animal que ya Aristóteles definió como Homo Ridens. Sin embargo, aun son muchas las incógnitas que siguen rodeando este curioso comportamiento, y algunas son tan fundamentales que afectan a todas las múltiples disciplinas que se acercan a su estudio. ¿Qué es? ¿Cómo funciona? ¿Qué la provoca? ¿Por qué y para qué, a lo largo de la evolución humana, se desarrolló? Estas cuestiones tan centrales siguen sin respuesta, y siguen dificultando la labor de los psicólogos, sociólogos, filólogos, 
lingüistas, filósofos y demás académicos que se han interesado por temas como el humor o la risa, muchos de los cuales hoy en día integran sus esfuerzos en la International Society for Humor Studies.

También los antropólogos, inevitablemente, han contribuido numerosas perspectivas y datos a este proyecto, y han tenido que vérselas con el complejo y escurridizo fenómeno. En este artículo me gustaría tratar el núcleo duro de esta problemática, desde el punto de vista de la antropología. Quisiera proponer un esquema teórico para orientar a los antropólogos interesados en el humor, que sirva de base para interpretar los diversos fenómenos culturales asociados a él. Trataré de distinguir entre el aspecto universal y psicológico de la risa, y su relación con los aspectos más específicos a cada cultura.

¿Es el humor algo universal o algo culturalmente específico? Se trata de una pregunta con mucha miga, ya que el humor se presenta en este sentido como una verdadera paradoja. Por un lado nada puede parecer más humano, y hay personajes, como el vagabundo de Chaplin y el ratón de dibujos animados Mickey Mouse, que han traspasado (casi) todas las fronteras culturales para convertirse en iconos de la cultura globalizada. Sin embargo y al mismo tiempo, el humor se presenta como la última barrera en el conocimiento de una cultura: si entiendes sus chistes, es que ya has llegado al corazón del sistema de símbolos y pensamientos de una sociedad, y ya nada te es desconocido. En estas páginas trataré de descifrar al menos parte de este misterio.

\section{La risa como emoción}

En primer lugar, quiero definir la risa como una emoción humana, un mecanismo psicológico común a todas las culturas e individuos que responde al "humor" definido aquí como cualquier estímulo de esta emoción. La risa se compone de un elemento afectivo subjetivo (la sensación de "hilaridad"), y de un elemento expresivo observable (las vocalizaciones y gestos que conocemos como "risa"), asociadas a cambios más sutiles a niveles neuroquímicos y fisiológicos. Y como en el caso de otras emociones, los componentes afectivos y expresivos de la risa se desencadenan a partir de una interpretación cognitiva de una realidad. La misma caída de un señor por unas escaleras puede provocar, según cómo se interprete, 
risa o dolor empático (viéndose como "humor" o como un infortunio muy serio). No es la caída en sí graciosa o no, sino la manera que cada persona la interpreta.

Debo notar que en las ciencias humanas y sociales se ha llegado a cuestionar la existencia de las emociones como fenómenos trans-culturales y biológicos. El psicólogo social de visión antropológica Otto Klineberg (1938) ya tomó nota de que en muchas sociedades, las expresiones emocionales se emplean de maneras que pueden parecernos insólitas. Citaba las expresiones hostiles de los Orokawa de Melanesia que empleaban para dar la bienvenida a los invitados a una fiesta, o la alegría mostrada por las mujeres samurai al escuchar la noticia de la muerte de sus maridos o hijos en plena batalla. Weston La Barre (1947) citó precisamente la risa como ejemplo de diversidad cultural:

\begin{abstract}
La risa es, en un cierto sentido, un variable geográfico. En un mapa del Sudeste del Pacífico, podrían dibujarse fronteras entre las zonas de "hilaridad papuana" y otras en las que reina una severidad cobuana y melanesia. En África, Gorer se fijó que el negro emplea la risa para expresar la sorpresa, el asombro, la vergüenza e incluso la incomodidad; no es necesariamente, o incluso frecuentemente, una señal de diversión. El significado que se le da a la "risa negra" se debe al error de suponer que símbolos similares tienen un significado idéntico. Así es que aunque se presente el mismo comportamiento fisiológico, sus funciones culturales y emocionales pueden ser distintas. De hecho, incluso dentro de la misma cultura, la risa de chicas adolescentes y la risa de presidentes de una empresa pueden ser cosas funcionalmente distintas ${ }^{1}$.
\end{abstract}

Más adelante, numerosos antropólogos propusieron que incluso emociones aparentemente similares en realidad difieren de manera significativa de una sociedad a otra en sus causas, fronteras e incluso su cualidad subjetiva, argumentando que "las pasiones son tan culturales como los artefactos" (Geertz, 1980: 124). Lutz (1988) y otros etnógrafos han llevado a cabo estudios de las vidas emocionales de pueblos concretos que parecen diferir significativamente de los nuestros.

Incluso en la psicología, hasta hace unos años, el paradigma dominante aceptaba la sorprendente propuesta (desde el punto de vista del "sentido común") que las emociones son el resultado de procesos cognitivos y no se asocian con procesos concretos biológicos y separables (ver Tomkins, 1981). En los años 80, se resumía el estado de la cuestión así:

\footnotetext{
${ }^{1}$ Éste y todos los demás textos citados en este artículo son mis traducciones. 
La psicología contemporanea coloca los sentimientos al final. El afecto es postcognitivo. Sólo aparece tras haberse procesado una cantidad considerable de información. Una reacción afectiva, como la atracción, el disgusto, la preferencia, la evaluación, o la experiencia del placer o la miseria se basa en un proceso cognitivo anterior en el que se llevan a cabo toda una variedad de discriminaciones del contenido y se identifican sus componentes, examinando su valor y dando peso a cada contribución. Una vez completada esta tarea analítica, la computación de los componentes puede generar una evaluación afectiva general (Zajonc, 1980: 151)

En contraste con el punto de vista de Charles Darwin (1902) y William James (1884) de que ciertas circunstancias específicas corresponden con respuestas emocionales específicas, la psicología escogió la teoría de W.B. Cannon (1927). Cannon propuso que una gran variedad de circunstancias estimulantes se asocian con una respuesta fisiológica única, que luego el sujeto interpreta como una u otra emoción. Según este punto de vista, tanto el temor como la ira provocan la activación del sistema nervioso simpatético mediante la descarga de la adrenalina, pero es la evaluación de las circunstancias del sujeto que le permite decidir qué emoción está experimentando.

Schachter y Singer (1962) llevaron a cabo un célebre experimento que se consideró prueba suficiente de la propuesta de Cannon, convirtiéndose en un paradigma clásico de la disciplina y una justificación de la falta de interés de la psicología académica en las emociones como fuerzas motivadoras. Los experimentadores inyectaron adrenalina a los sujetos y les colocaron en situaciones que debían provocarles alegría o ira. Confirmando la predicción de los experimentadores, los sujetos actuaron y se sintieron alegres en el primer contexto y enfadados en el segundo. El hecho de que distintas emociones puedan compartir ciertas características fisiológicas no demuestra, sin embargo, que no existan diferencias extracognitivas entre ellas. Aparte de los problemas metodológicos que más adelante se criticaron en el experimento de Schacter y Singer, y de los resultados bastante débiles y en algunos casos contradictorios de la prueba, es posible que los sujetos que participaron experimentaran emociones bien distintas, que la adrenalina sencillamente amplificó. Sin embargo, las conclusiones de Schachter y Singer se aceptaron casi sin excepción durante más de dos décadas en un panorama psicológico dominado por el cognitivismo (Tomkins, 1981: 310-312).

A partir de los años ochenta, sin embargo, comenzaron a surgir nuevas pruebas de la separabilidad fisiológica de las emociones. Se comprobó en el laboratorio que distintas emociones producían patrones específicos de medidas 
como la conductividad de la piel, el ritmo cardíaco o la temperatura de la cabeza (Ekman, Levenson, and Friesen, 1983 y 1990; Stemmler, 1989). Un estudio transcultural de Paul Ekman (Ekman y Friesen, 1971) provocó un debate en las ciencias sociales al demostrar que muchas expresiones emocionales se heredan al menos en parte, ya que son interpretables en culturas muy distantes. Se le presentó a miembros de los Fore del Sur, una comunidad aislada de Nueva Guinea, una serie de fotos de estudiantes universitarios norteamericanos posando con diversas expresiones emocionales: ira, disgusto, temor, etc. Los Fore fueron capaces de interpretar estas expresiones correctamente (relacionándolas con unas "historietas emocionales") y cuando los investigadores fotografiaron a los Fore posando de manera similar, sujetos occidentales fueron igualmente capaces de interpretar las emociones mostradas. Otras pruebas que también plantearon dudas respecto al paradigma dominante fueron las de los niños que nacen ciegos y sordos, que son incapaces de aprender las expresiones emocionales de los demás, y que sin embargo se comportan de maneras fácilmente interpretables por cualquier persona (Eibl-Eibesfeldt, 1973). Finalmente, los avances de la neuropsicología acabaron por demostrar que distintas emociones efectivamente se corresponden con sutiles cambios cerebrales, aun poco conocidos pero claramente diferenciables (Kolb, y Wishaw, 2003). Hoy en día, el paradigma sobre las emociones ha cambiado radicalmente en la psicología, y no sólo se acepta que existan como tales, sino que cumplen un importante rol en la motivación humana. En los últimos años, incluso se ha revalorizado el papel de las emociones positivas, aun más relegadas, si cabe, al trastero de la psicología académica durante el último siglo, y entre ellas la risa (Seligman, 2002). De hecho, el reciente libro The Psychology of Humor de Rod Martin (2006), el primer libro de texto serio que reúne toda la investigación empírica sobre el humor, define la risa claramente como emoción positiva, y parece ésta actualmente la postura más extendida en este campo.

\section{La expresión de la risa y de otras emociones}

¿Qué aspectos de la risa, entonces, son universales, y cuáles son modificables culturalmente? Antes de responder a esta pregunta debo precisar la diferencia entre la emoción en sí y su expresión. Desafortunadamente, en castellano no existe una 
palabra de uso cotidiano que identifique este fenómeno emocional sin ambigüedades, dado que "risa" indica también, e incluso principalmente, la expresión de la emoción: los sonidos y gestos que la acompañan. Podríamos emplear la palabra "hilaridad" (pero a mí me parece un tanto forzada), "diversión" (pero esta tiene otras connotaciones que van más allá del humor) o "humor" (que también tiene múltiples significados). Por lo tanto, hablaré de "risa" y de "expresión de la risa", esperando que en el contexto el significado sea claro. En cualquier caso es imprescindible tener clara esta distinción.

La expresión de la risa, como en el caso de otras emociones, es controlable, al menos hasta cierto punto. Puede modificarse su intensidad, su forma y su duración. Por este motivo, hay diversos investigadores que han tratado la expresión de la risa como un signo comunicativo (Martineau, 1972). A diferencia de los signos lingüísticos, sin embargo, el control de las expresiones emocionales es limitado. Es una habilidad aprendida y que no siempre tiene éxito -es posible reír cuando uno no quiere, o fracasar en el intento de reír de manera convincente cuando la situación no resulta graciosa. Por este motivo, las expresiones emocionales son para los observadores sociales más fiables que los signos lingüísticos.

¿Cómo puede entonces la cultura de una sociedad influir sobre la risa? En primer lugar, puede influir directamente sobre el funcionamiento de la emoción misma, aunque el mecanismo sea universal. Las emociones son idénticas en todo el mundo, pero la manera de interpretar la realidad que provoca su desencadenamiento puede variar de un momento a otro y de una persona a otra $-\mathrm{y}$ mucho más de una cultura a otra. La misma máscara que aterroriza a un niño pequeño puede parecerle divertida a un adulto. El descubrimiento de un tumor de dimensiones y forma insólita podría provocar miedo al paciente, llanto en su familia y curiosidad científica en el cirujano. Una temperatura externa que haría sonreír a un Inuit haría fruncir el ceño a un marroquí (y vice-versa). Los espectáculos sangrientos del Coliseo que divertían a los ciudadanos de Roma inspirarían horror e indignación entre los italianos modernos. Estas reacciones opuestas reflejan divergencias en las actitudes, creencias e interpretaciones, y no en los mecanismos emocionales mismos. Un grupo concreto puede desarrollar también un umbral específico para una emoción que sea mayor o menor que la de otros, al aprender ciertos estilos de interpretación en las circunstancias relevantes. En sociedades en las que a los 
individuos se les conceden todo tipo de derechos (incluidos los de la propiedad privada y exclusiva), es más fácil que florezca la ira (gracias a las violaciones inevitables de estos derechos). Diferencias más generalizadas en los estilos emocionales pueden reflejar aspectos culturalmente variables de la personalidad, como el énfasis en la individualidad y la independencia de cada actor social (Oatley y Jenkins, 1996: 42-43). Sin duda, los antropólogos pueden dibujar "mapas emocionales" de la geografía humana y enlazar las fuentes de las reacciones emocionales de diversos pueblos a las estructuras culturales y sociales que los articulan.

Una segunda vía de influencia cultural se refiere a las reglas sociales y los objetivos individuales que afectan la expresión de una emoción. La expresión emocional depende en parte de las normas que rigen la comunicación en la situación concreta (¿esta expresión es aceptable o requerida aquí y ahora?) y también de las metas del actor en cuestión (¿me interesa expresar esta emoción aquí y ahora?). En un conocido experimento (Ekman, 1972; Friesen, 1972), se comprobó que (1) por un lado, tanto un grupo de universitarios japonés como otro norteamericano mostraban expresiones de disgusto al ver un documental con escenas de cirugía, pero que (2) por el otro lado, los primeros mostraban menos disgusto si sabían que se les estaba observando. El nivel general de emotividad considerado "normal" puede también variar entre una sociedad y otra. En las palabras de Mary Douglas (1971), "los umbrales de tolerancia de la relajación y el control del cuerpo se establecen socialmente". Estos umbrales afectarán, a su vez, la intensidad y el tipo de expresiones emocionales adecuadas en general y en contextos específicos.

Ciertos usos de expresiones emocionales y de los estímulos que los provocan pueden también institucionalizarse o volverse más comunes en una sociedad concreta, ya sea por accidente histórico o para obtener resultados culturalmente valorados. En una sociedad militarizada, por ejemplo, las instituciones de socialización y control pueden orientarse hacia la supresión del miedo y el fomento y canalización de la ira (ver David, 1988). Por otro lado, en sociedades donde las consecuencias violentas y antisociales de la ira podrían amenazar la supervivencia o 
los valores fundamentales, la expresión de esta emoción puede desaparecer casi por completo. (Briggs, 1970)².

Finalmente se presenta la cuestión de las interpretaciones culturales de las emociones. Como cualquier otro aspecto del entorno, las reacciones emocionales son el objeto de clasificación y definición en cada sociedad. Del mismo modo que los Inuit distinguen numerosas variedades de nieve, los Tahitianos tienen 47 vocablos que se refieren a la ira (Levy, 1984). Por otro lado, el contenido de los conceptos emocionales y las fronteras que los separan pueden variar enormemente. La emoción que los Ifaluk llaman "metagu" integra nuestras ideas de "temor", "ansiedad" y "vergüenza" (Lutz, 1982), mientras nuestro "miedo" se refiere en parte a una emoción que los Tahitianos consideran algo específico y distinto, la emoción de lo "inexplicable", una sensación que según ellos hace que se nos pongan los pelos de punta cuando observamos algo aparentemente "imposible" (Levy, 1984). Sin embargo, puede argumentarse que estas diversas teorías de las emociones o "emocionologías" (Stearns y Stearns, 1988) se refieren a una realidad básica de predisposiciones emocionales comunes a todos los seres humanos, sobre las cuales estos conceptos específicos se basan:

A pesar de las peculiaridades culturales en las relaciones y los sentidos asociados a los términos emocionales tahitianos, no me costaba reconocer, digamos, ri'ari'a como "miedo," riri como "ira," hina'aro como "deseo," 'oa'oa como "felicidad," ha'ama como "vergüenza." O sea, si una emoción se reconocía y recibía un nombre, la "tendencia central" del concepto parecía ser universalmente humana (Levy, 1984)

Estas palabras nos dan la clave para entender la existencia de estas "emocionologías" a pesar de que ya es difícil dudar de que existan numerosas emociones básicas comunes a toda la especie humana, como los psicólogos ya han demostrado ampliamente.

\section{El elemento común del humor}

Quizás el mayor escollo a la hora de entender la risa como una emoción universal, y en general para entender la risa, es la falta de una teoría generalmente aceptada

2 Probablemente existan diversos estilos y habilidades de control emocional, algunas más "superficiales", que tratan de suprimir o elaborar las expresiones visibles, y otras más "profundas" que traten de eliminar o generar la emoción en sí (Ver Hochschild, 1979). 
que explique cuáles son sus causas. ¿Qué provoca la risa? ¿Tienen algo que ver los chistes, el humor del Quijote, los juegos de los niños y las cosquillas? Los filósofos y científicos que han tratado de dar respuesta a estas preguntas siguen sin ponerse de acuerdo, aunque se han propuesto cientos de ideas para resolver el enigma. Este problema quizás explique como es que la risa apenas suele citarse en libros de texto sobre las emociones, o incluso en libros sobre psicología positiva (ver Oatley and Jenkins, 1996, y Seligman, 2002). Aparte de esta causa identificable, tan difícil de definir, la risa cumple todos los requisitos de Paul Ekman que permiten identificar una emoción "básica" (1992).

En mi opinión, sí existe un elemento común a las diversas tipologías y géneros humorísticos, o al menos una serie de causas universales. De hecho, en mi tesis doctoral (Jáuregui, 1998) desarrollé una teoría causal para explicar toda la variedad del humor, basada en la teoría dramatúrgica de la interacción social de Erving Goffman (ver también Jáuregui 2003 y Jáuregui 2007). Esta teoría en mi opinión pertenece a una "quinta escuela" de teorías (entre las que se encuentran la de Aristóteles, Platón, Bergson y otros) que ofrece una alternativa a las más conocidas escuelas de la Incongruencia, la Superioridad, la Catársis y el Juego. En cualquier caso, no hace falta aceptar ni mi teoría ni cualquiera de las alternativas para observar que el mismo tipo de eventos que en la sociedad occidental se consideran "graciosos", también hacen reír en las culturas más dispares, y en todas las épocas conocidas.

Los viajeros, misionarios y etnógrafos que han tenido contacto con toda la impresionante variedad de sociedades humanas parecen haber reconocido la expresión de la risa como tal (o sea, como señal de una hilaridad interior provocada por causas similares), en todas las esquinas del planeta. Noticias sobre la risa y sus fuentes aparecen aquí y allí en la literatura etnográfica, y tienden a confirmar la universalidad del humor. Los textos de Marshall (1961) sobre los bosquímanos !Kung, una sociedad nómada y cazadora en las antípodas culturales de nuestra propia sociedad urbana y cosmopólita, suenan muy familiares. Marshall cuenta que los !Kung están siempre hablando cuando se encuentran en grupo, siendo "el pueblo más locuaz que conozco", y comenta que la conversación se alterna frecuentemente con "chillidos de risa" (p. 232): 
Se ríen de las pequeñas desventuras de otras personas, como cuando los leones se comen la carne de otro, y chillan de la risa cuando alguien hace algún comentario sexual especialmente insultante en la relación bromista... Los hombres y las mujeres que tienen una relación bromista se insultan en clave de humor y también resaltan faltas verdaderas o comentan episodios reales que ponen en ridículo al otro. Todos se unen en una gran risotada a costa de la víctima (p. 233-35)

Esta descripción de Marshall podría aplicarse (¡aparte de la referencia a los leones!) a cualquier grupo de amigos en la sociedad occidental.

Incluso en relación con algunas de las comunidades más aisladas, y en ausencia de un lenguaje común, los etnógrafos pueden inmediatamente compartir la risa con individuos de raíces culturales radicalmente distintas:

En una ocasión, subí a las montañas hasta la zona más alejada del territorio Dowayo. Muchos de los niños nunca habían visto a un hombre blanco y comenzaron a gritar de terror hasta que los mayores les tranquilizaron y les explicaron que se trataba del jefe blanco de Kongle. Todos nos reímos con buen humor de su miedo y fumamos juntos (Barley, 1983: 54)

Los estudios etnográficos del humor revelan que las historias cómicas tradicionales se basan en las mismas pretensiones infladas y las mismas caídas en el ridículo que pueden encontrarse en las comedias de Cervantes, Moliere, Shakespeare, Boccaccio o incluso Santiago Segura. Pierre Clastres (1974) analizó dos mitos de los Indios Chulupi del Chaco Paraguayo que provocan grandes risas cada vez que se cuentan. Cada uno de estos trata sobre figuras culturalmente importantes -el chamán y el jaguar- que fracasan a la hora de cumplir las exigencias más básicas de sus roles. En un mito, se le llama al chamán para que cure la fiebre de una niña, y cada una de sus acciones, como en una película de Peter Sellers, es una demostración de incompetencia: pide la ayuda de otros chamanes (una medida normalmente reservada para casos desesperados, que aquí se aplica para una trivialidad); baila con las mujeres (cuando debería bailar sólo); trata de seducir a las mujeres (implicando una falta de la concentración necesaria para realizar su trabajo); descubre haber olvidado su tabaco (la medicina indispensable del chamán); su espíritu-asistente es una burra (considerado un animal ridículo, estúpido y terco) en vez de la habitual serpiente o pájaro; incluso llega a caerse torpemente en un arbusto espinoso.

Una amplia variedad de géneros humorísticos parecen comunes a lo largo y ancho de la humanidad, incluidas la rotura de tabúes sexuales y escatológicos, el insulto verbal, la violencia física, la burla, el "cotilleo", las payasadas y las 
imitaciones satíricas (Apte, 1983). A continuación cito como ejemplo una descripción de las bromas típicas observadas en las "relaciones bromistas" o joking relationships, un tipo de relación que se ha descrito en diversas sociedades de todas las principales áreas geográficas:

Tomar el pelo, connotaciones sexuales, insultos en broma, comentarios obscenos sobre los órganos y las capacidades sexuales, el juego, el ingenio lingüístico, etc... También son importantes las nociones culturales de las manifestaciones de la personalidad o del comportamiento, etc, que se consideran incongruentes, escandalosos o pervertidas. (Apte, 1983: 186)

La descripción de los payasos rituales en diversas culturas de nuevo sugiere que las diferencias en el sentido del humor de una u otra sociedad son más una cuestión de gustos, modas y énfasis que de sustancia:

\begin{abstract}
Los payasos eran extravagantes, sus caras pintadas medio de blanco, medio de negro. Se vestían con basura o trapos viejos y hablaban con gritos atiplados medio en fulani, medio en dowayo, chillando obscenidades y tonterías. "iEl coño de la cerveza!" gritaban. El público explotaba de la risa. Exhibían sus posteriores, produciendo flatulencias extraordinarias mediante algún mecanismo que no conseguí averiguar. Trataban de copular los unos con los otros. Se divertían muchísimo conmigo. Me "sacaban fotos" a través de un cuenco roto, "escribían apuntes" sobre hojas de plátano. Yo trataba de devolverles la broma. Cuando me pedían dinero, yo les entregué solemnemente la chapa de una botella. (p. 83-84)
\end{abstract}

El yanqui de Connecticut que en la novela de Mark Twain viaja misteriosamente a la corte del Rey Arturo se sorprende de la falta de novedades en el humor moderno, comparado con las bromas de un bufón medieval:

Creo que nunca había oído tantos chistes viejos en mi vida... Me pareció peculiar estar ahí, 1300 años antes de mi propio nacimiento y escuchar de nuevo los mismo chascarrillos pobres y gastados que ya me habían aburrido cuando fui un niño 1300 años después. Prácticamente me convenció que no existe la posibilidad de inventar un chiste nuevo (citado en Mintz, 1983: 133)

Para apoyar esta observación literaria, a menudo reflejada también en la ideas del folklore cotidiano de que un chiste es "viejísimo", Larry Mintz ha encontrado impresionantes parecidos entre cómicos tan distantes como los Hermanos Marx y las tropas de la Commedia del Arte del siglo XVI, o entre los textos ingeniosos de las comedias griegas y la rutina cómica más célebre de Abbott y Costello:

[de los Cíclopes, Euripides, 400 AC]

AIBR. Revista de Antropología Iberoamericana. www.aibr.org

Volumen 3, Número 1. Enero-Abril 2008. Pp. 46-63

Madrid: Antropólogos Iberoamericanos en Red. ISSN: 1695-9752 


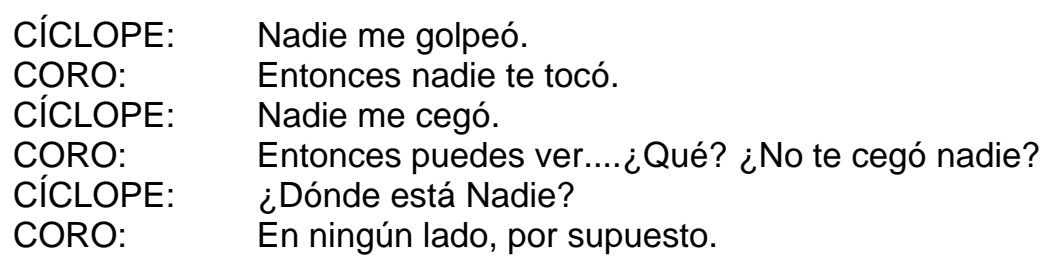

[del diálogo absurdo de Abbott y Costello sobre los jugadores -en primera, segunda y tercera base- del nuevo equipo de beisbol de Abbott]

ABBOTT: $\quad$ A ver, Quién está en la primera base, Qué en la segunda y en la tercera Nolosé.

COSTELLO: Entonces ¿sabes cómo se llaman los jugadores?

ABBOTT: Sí.

COSTELLO: Vale, ¿Quién está en la primera?

ABBOTT: Sí.

COSTELLO: Digo el tipo que juega primera.

ABBOTT: Quien.

COSTELLO: El tipo en primera base.

ABBOTT: Quien.

COSTELLO: El de la primera.

ABBOTT: ¡Quién!

COSTELLO: El que juega en primera.

ABBOTT: iQuién está en la primera!

COSTELLO: Pero, ¿por qué me lo preguntas a mí? (Mintz, 1983: 133)

Ejemplos más espontáneos y cotidianos de todo el mundo refuerzan esta impresión de universalidad. Muchas anécdotas de este tipo recopilados por viajeros y etnógrafos occidentales fueron provocados por los visitantes mismos, que inevitablemente violaban sin querer las normas básicas del comportamiento social:

Los nativos de Sumatra, según una autoridad, tienen bailes muy lentos que los europeos consideran ridículos. Sin embargo, y curiosamente, ellos creen que nuestros bailes tradicionales son "totalmente absurdos".

Según cuenta un viajero, [en Borneo] las niñas hacían repetir a los europeos frases de su lenguaje, y se echaban a reír abiertamente "o de nuestra pronunciación o de las cosas cómicas que nos habían hecho decir".

Los habitantes de la Tierra del Fuego, aunque pasaban gran parte del tiempo en el agua, no tenían el concepto del lavarse; por lo tanto, "cuando los europeos aparecieron por primera vez entre ellos, la vista de un hombre lavando su cara les parecía tan irresistiblemente divertido que explotaban a reír a carcajadas".

Un maestro de los aborígenes australianos trató de explicar a uno de ellos la doctrina de la inmaterialidad y la inmortalidad del alma. Después descubrió que su pupilo se había ido de su lección para reír a gusto del absurdo de la idea "de un hombre viviendo y dando vueltas sin brazos, ni piernas, ni boca para comer (Sully, 1902: 240; 238; 243)

Estos ejemplos sugieren que, independientemente de qué teoría empleemos para explicar la variedad del humor, las situaciones más típicas que provocan la risa en 
España, Francia o Estados Unidos no son fundamentalmente distintas de las que resultan graciosas en sociedades mucho más alejadas culturalmente.

\section{Variabilidad cultural}

No obstante, es evidente que el carácter específico de los estímulos humorísticos varía tan radicalmente como la cultura misma, y de hecho en paralelo con ella. Para explicar estas diferencias sí es necesario tomar como referencia alguna teoría causal del humor, y adoptaré por lo tanto la mía, aunque sería posible traducir estos mismos argumentos en otras claves teóricas. Según la teoría dramatúrgica, el estímulo de la risa es la percepción de que otro actor social ha incumplido su rol dentro del teatro de la vida cotidiana --el papel que él mismo se ha creado o que la sociedad le impone. Siguiendo esta clave teórica, puede decirse que lo que se considera "gracioso" en una cultura se encuentra en directa relación con las expectativas sobre los roles que son relevantes en esta sociedad concreta, incluido el rol más general que podemos denominar el de una "persona normal". Lavarse la cara, para los occidentales con sus ideas de higiene, es un rito diario fundamental, mientras que para los nativos de la Tierra del Fuego resultaba un absoluto disparate. En la Esparta antigua, una sociedad militar donde la demostración del coraje era una de las claves del rol social de los varones, "la categoría más prominente de los bufones... la constituían los "tembladores" (tresantes)":

La cobardía en la batalla se castigaba en Esparta con una serie de penalizaciones legales... Además de las sanciones legales, el castigo de los "tembladores" exigía ciertas normas que en definitiva transformaban las víctimas en miserables payasos: "Cualquier viandante los puede golpear si quiere. Además, deben ir por la calle con un aspecto penoso, vestidos con túnicas con parches de colores, con la mitad de la barba afeitada y la otra mitad sin afeitar (David, 1988: 14)

Un aspecto de la teoría dramatúrgica (que comparte con otras teorías del humor) se refiere a la distancia emocional que la risa requiere: si existen elementos de la situación que provocan otras emociones, como la tristeza, la ira o la curiosidad, estas pueden reducir o suprimir la apreciación del humor. Por lo tanto, las sensibilidades distintas que existan en una cultura hacia diversos tipos de desgracia u obscenidad pueden condicionar también las reacciones hacia estímulos potenciales del humor. Marshall cuenta sobre los !Kung que "se ríen cuando el 
hombre cojo, Kham, se cae, y no le ayudan a levantarse (1961, p.231). Esta reacción parece incomprensible ("cruel") desde el punto de vista de los occidentales, que sin embargo no dudan en partirse de la risa disfrutando de las caídas ficticias de los cómicos cinematográficos. Debe interpretarse en el contexto de una sociedad nómada donde la supervivencia depende entre otras cosas de movilidad independiente de cada persona. En su mundo del desierto, una excesiva "empatía" con las personas discapacitadas probablemente pondría en peligro la supervivencia de toda la colectividad.

Ciertos géneros del humor y las reglas sobre cuándo y cómo deben exhibirse también pueden ser culturalmente específicos, aunque la variabilidad queda limitada, de nuevo, por la intención común de provocar una reacción emocional universal. Los carnavales y los bufones de la Edad Media pueden analizarse en relación con la estructura social y la cultura de la Europa feudal, del mismo modo que las sitcom televisivas y la comedia posmoderna guarda relación con características de nuestra propia sociedad.

El desarrollo de técnicas artísticas y de medios de comunicación también tiene una influencia importante sobre las formas del humor. El desarrollo de la prensa, de la radio, de la televisión y de Internet han transformado el contenido de la comedia y ampliado sus posibilidades en los últimos dos siglos. La historia y la etnografía de la literatura y el teatro también incorpora todo el catálogo de géneros humorísticos. Un ejemplo extremo de la variabilidad cultural, en este sentido, de nuevo nos llega de los bosquímanos !Kung:

No inventan cuentos. Dijeron que no tienen interés en escuchar cosas que no son ciertas, y se preguntan cómo es que pueden interesar a nadie (Marshall, 1961: 233)

El repertorio humorístico posible, en una sociedad que no conoce la ficción, parecerá extremadamente pobre a cualquier occidental. Sin embargo, como ya hemos citado anteriormente, los estímulos humorísticos de los !Kung no se diferencian mucho de situaciones y anécdotas que también a nosotros nos hacen reír.

Como cualquier emoción, la risa queda condicionada en cualquier cultura por normas específicas sobre cómo y cuándo puede expresarse, y por los motivos de los actores concretos, según los significados y valores de la sociedad. En 1902, Sully ya identificó el contraste entre la expresión de las emociones y el substrato emocional 
verdadero como un posible obstáculo a la hora de estudiar la risa. Citó la descripción de un misionero africano que contaba que los nativos a menudo mostraban un aspecto solemne ante los errores o accidentes de los europeos, pero que luego "se divierten a su costa cuando éste se ha ido, y de hecho, incluso en su presencia si saben que no entenderá sus palabras". (p. 221). Más recientemente, Chapman (1983) ha observado la misma estrategia de ocultación de la risa en el laboratorio experimental. El estilo y nivel general de risa aceptable puede variar también de una sociedad a otra:

Sabemos que algunas tribus tienden a ser solemnes y poco proclives a la risa. Otras ríen fácilmente. Los pigmeos caen al suelo y agitan sus piernas en el aire, jadeando y agitándose con una risa que les posee por completo (Douglas, 1971: 387)

Ciertos usos del humor y de la risa pueden también institucionalizarse o volverse más comunes en una sociedad concreta, en un grupo o en un entorno, ya sea por sencillo accidente histórico, o para obtener algún efecto especialmente deseable. En las sociedades pequeñas de nómadas, por ejemplo, en las que la cooperación de cada miembro puede ser crucial para la supervivencia común, el uso del humor y de la risa para resolver disputas es habitual, concretándose a menudo en la figura de un "payaso" del grupo que sirve de mediador, juez y agente de la paz (Roberts, 1979: 88). En otras sociedades en las que el conflicto no resulta igualmente amenazante, el potencial pacifista del humor puede permanecer menos institucionalizado. Del mismo modo que los "risoterapeutas" de la actualidad enseñan a sus clientes a desarrollar su sentido del humor, una cultura que reconoce los beneficios de la risa podría fomentar también el empleo del humor por estos motivos. De hecho, este es el sentido que el psicólogo William Fry atribuye, por ejemplo, a los festivales de invierno que son habituales en sociedades que habitan las zonas del planeta con inviernos largos y oscuros (Fry, 2004).

En relación a las interpretaciones culturales de la risa en sí, y su relación con otros aspectos de las emociones y el comportamiento, conozco pocos estudios concretos -el de David's (1988) sobre la risa en Esparta es un ejemplo. En cualquier caso, no hay duda de que las clasificaciones y descripciones de las causas, características, fisiología y efectos de la risa varían también enormemente de una sociedad a otra. La gran variedad de teorías que a lo largo de la historia de la filosofía y de la ciencia han tratado de explicar el humor ya constituyen una variada 
gama de posibilidades. Centrándonos sólo en el lugar de la risa en relación a las otras emociones, encontramos que se ha propuesto como similar al deleite o el placer (Sully, 1902; Morreall, 1982), al disgusto (McDougall, 1913, citado en Grieg, 1923), al dolor (placentero o lúdico) (Platón, 1961; Eastman, 1921), al "sentimiento fuerte de casi cualquier tipo" (Spencer, 1860) y a la agresión (Feinberg, 1978). Koestler (1964) la identifica como un elemento de un trío que completan el descubrimiento científico y el placer estético. Baillie (1921) y Hobbes (1651) lo ven como un complemento al llanto y Bergler (1956) como opuesto al miedo "del propio masoquismo interno". Mi propia teoría también coloca la risa dentro de una particular "emocionología", concretamente como un complemento de la vergüenza social (la risa y la vergüenza reaccionan al mismo tipo de situaciones, vistas desde dos puntos de vista distintos: la persona que hace el ridículo y el que la observa).

\section{Conclusión}

Una visión amplia de los estudios psicológicos y antropológicos sobre la risa y el humor permiten distinguir entre los aspectos universales y aquellos que son culturalmente variables. La risa puede definirse como un mecanismo emocional que funciona según las mismas pautas en todos los seres humanos, pero la naturaleza social de la evaluación cognitiva que la provoca tiene como resultado una enorme variedad aparente en sus causas, y en las instituciones y géneros artísticos que en cada cultura se desarrollan en torno a ellas. Por otro lado, la expresión de la risa misma es también culturalmente variable, así como las teorías populares sobre su funcionamiento.

\section{Bibliografía}

Apte, M. (1983) Humour Research, Methodology, and Theory in Anthropology, A Handbook of Humour Research, Vol. I, P.E. McGhee y J.H. Goldstein, eds., Nueva York: Springer-Verlag.

Baillie, J.B. (1921) Laughter and Tears. Studies in Human Nature, pp.254-293. Londres: Bell and Sons.

Barley, N. (1983) The Innocent Anthropologist: Notes From a Mud Hut. Londres: Penguin.

Bergler, E. (1956) Laughter and the Sense of Humor, Nueva York: Intercontinental Medical Book Co.

Briggs, J. L. 1970. Never in Anger. Cambridge: Harvard Univ. Press 
Cannon W.B. (1927). The James-Lange theory of emotions. American Journal of Psychology, 39:115-124.

Chapman, A. J. (1983) Humor and Laughter in Social Interaction and Some Implications for Humor Research, A Handbook of Humour Research, Vol. I, P.E. McGhee and J.H. Goldstein, eds., Nueva York: Springer-Verlag.

Clastres, P. (1989) Society Against the State: Essays in Political Anthropology. Traducido por Robert Hurley and Abe Stein. Nueva York: Zone Books.

Darwin, C. (1890/1989) The Expression of the Emotions In Man and Animals, The Works of Charles Darwin, P.H. Barrett and R.B. Freeman, eds., Londres: William Pickering.

David, E. (1988) Laughter in Spartan Society. En Classical Sparta: Techniques Behind her Success. Anton Powell (ed.).

Douglas, M. (1971) Do Dogs Laugh, Journal of Psychosomatic Research, Vol. 15.

Eastman, M. (1921) The Sense of Humor, Nueva York: Charles Scribner's Sons.

Eibl-Eibesfeldt, I. (1975) Ethology: The Biology of Behavior, 2nd ed., NY: Holt, Rinehart and Winston.

Ekman, P. (1972) Universals and cultural diferences in facial expressions of emotion. In Cole (Ed.) Nebraska Symposium on Motivation, 1971 (pp. 207-83). Lincoln, NE: University of Nebraska Press.

Ekman, P. (1978) Facial Signs: Facts, Fantasies, and Possibilites, Sight, Sound and Sense, Thomas A. Sebeok, ed., Bloomington: Indiana University Press.

Ekman, P. (1980) The Face of Man. Nueva York: Garland.

Ekman, P. y W.V. Friesen (1971) Constants across culture in the face and emotion. Journal of Personality and Social Psychology, 17, 124-29.

Feinberg, L. (1978), The Secret of Humor, Amsterdam: Rodop.

Fry, W. (2004) El humor, la biología y la psicoterapia. El humor y el bienestar en las intervenciones clínicas. Salameh, W. y Fry, W. (eds). Bilbao: Desclee de Brouwer.

Geertz, C. (1980). Negara: The Theater State in Nineteenth-Century Bali. Princeton: Princeton University Press.

Grieg, J.Y.T (1923/1969), The Psychology of Laughter and Comedy, Nueva York: Cooper Square.

Hobbes, T. (1640/1966) Human Nature, The English Works of Thomas Hobbes of Malmesbury, Sir William Molesworth, Ed., Vol. III, Londres: John Bohn.

Hochschild, A. R. (1979) Emotion Work, Feeling Rules, and Social Structure, American Journal of Sociology, Vol. 85, pp. 551-575.

James, W. (1890/1950) The Principles of Psychology, Nueva York: Dover.

Jáuregui, E. (2007) El sentido del humor: manual de instrucciones. Barcelona: RBA.

Jáuregui, E. (2003) La comedia humana: una nueva teoría psico-sociológica de la risa y el humor. Miscelanea Comillas, 61, 563-83.

Klineberg, O. 1938 Emotional expression in Chinese literature, Journal of Abnormal and Social Psychology, 33: 517-520.

Koestler, A. (1964) The Act of Creation. Londres: Hutchinson and Co.

Kolb, B., y Wishaw, I.Q. (2003). Fundamentals of Human Neuropsychology (5th edition). NY: Freeman.

La Barre, W. Cultural Basis of Emotions and Gestures, Journal o! Personality, 16 (1947), pp. 49-68

Levy, R. I. 1984. Emotion, knowing, and culture. Pp. 214-237 in Culture Theory: essays on mind, self, and emotion., edited by R. Shweder and R. LeVine. Cambridge, UK: Cambridge University Press.

Lutz, C., 1982. The domain of emotion words on Ifaluk. Am. Ethnol. 9, pp. 113-128

Lutz, C. (1988) Unnatural Emotions: Everyday Sentiments on a Micronesian Atoll and Their Challenge to Western Theory. Chicago: UCP.

Marshall, L. (1961) Sharing, Talking, and Giving: Relief of Social Tensions Among !Kung Bushmen. Africa. 31, 3.

Martin, R. (2006) The psychology of humor. NY: Academic Press. 
Martineau, W. H. (1972) A Model of the Social Functions of Humor, The Psychology of Humor, J.H. Goldstein and P.E. McGhee, eds., Nueva York: Academic Press.

Mintz, L.E., (1983) Humour and Popular Culture in McGhee and Goldstein (eds.) A Handbook of Humour Research, Vol. II, P.E. McGhee and J.H. Goldstein, eds., Nueva York: Springer-Verlag.

Morreall, J. (1982) A New Theory of Laughter, Philosophical Studies, 42: 243-54.

Oatley, K., y Jenkins, J. (1996) Understanding emotions. Cambridge, Mass.: Blackwell Publishers, Inc.

Platón (1961) 'Philebus', Plato: The Collected Dialogues. E. Hamiton and H. Cairns, eds. Princeton, NJ: Princeton University Press.

Roberts, Simon (1979) Order and Dispute. London: Penguin.

Schachter, S., y Singer, J., Cognitive, Social, and Physiological Determinants of Emotional State, Psychological Review, 1962,69, 379-399.

Seligman, M. (2002) Authentic Happiness. Londres: Nicholas Brealey.

Spencer, H. (1891/1966) The Physiology of Laughter, The Works of Herbert Spencer, Vol. XIV: Essays, Scientific, Political and Speculative (Vol. II), Osnabruck: Otto Zeller.

Stearns, C.Z. y P.W. Stearns. 1988. Emotion and social change. Nueva York: Holmes and Meier.

Stemmler, G. (1989). The autonomic differentiation of emotions revisited: Convergent and discriminant validation. Psychophysiology, 26, 617-632

Sully, J. (1902) Essay On Laughter, Londres: Longmans, Green and Co.

Tomkins, S. (1981). The quest for primary motives: Biography and autobiography of an idea. Personality and Social Psychology, 41:306-329.

Zajonc, R.A., (1980) Feeling and Thinking: Preferences Need No Inferences, American Psychologist , 35, pp. 151-175. 